\title{
ENTRAMADOS DE INTERDEPENDENCIAS, CUIDADOS Y AUTONOMÍA EN SITUACIONES DE DIVERSIDAD FUNCIONAL
}

\section{Frameworks of Interdependence, Care and Autonomy in Situations of Functional Diversity}

\author{
María Pía Venturiello \\ CONICET. Instituto de Investigaciones Gino Germani. Universidad de Buenos Aires \\ Carmuca Gómez Bueno \\ Universidad de Granada \\ María Teresa Martín Palomo* \\ Universidad de Almería \\ Centro de Estudio de las Migraciones y las Relaciones Interculturales (CEMyRI)
}

\section{Palabras clave}

Situación de dependencia Auto-cuidados Autonomía Subjetividad Vulnerabilidad

\section{Keywords}

Dependency situation

Self-care

Autonomy

Subjectivity Vulnerability

\begin{abstract}
RESUMEN: Entender la sociedad como entramados de interdependencias, y la autonomía personal como capacidad de decisión y acción, se opone al sentido que algunas organizaciones atribuyen a las categorías autonomía y dependencia. En España, la producción e imposición de etiquetas administrativas al amparo de la Ley de Promoción de la Autonomía Personal y Atención a las Personas en Situación de Dependencia (Ley 39, 2006), convierte a dichas categorías en los dos polos de un continuo. Frente a este marco institucional, el artículo analiza los modos en que mujeres con diversidad funcional, oficialmente catalogadas como «severas" o "grandes dependientes" y que cuentan con el servicio de ayuda a domicilio, organizan su vida diaria, sus redes de interdependencias y la capacidad de agencia que despliegan, a partir del análisis de sus relatos de vida. Desde una perspectiva del cuidado se exploran posibles líneas para resignificar la dependencia y la autonomía, a partir de los modos en que estas mujeres ejercen la auto-determinación, la afirmación personal y el auto-cuidado, y los impactos de estos en la subjetividad. De ello se concluye que la determinación de sí mismas para el ejercicio del autocuidado es fundamental para la redefinición de su autonomía.
\end{abstract}

ABSTRACT: Understanding society as networks of interdependencies, and personal autonomy as decision and action capacity, is opposed to the sense that some organizations attribute to the categories of autonomy and dependency. In Spain, the production and imposition of administrative labels under the Law of Promotion of Personal Autonomy and Attention to People in Dependency Situation (Law 39, 2006), turns these categories into two poles of a continuum. Faced with this institutional framework, the article analyzes the ways in which women with functional diversity, officially classified as "severe" or "large dependents" and who have the home-care help service, organize their daily lives, their networks of interdependencies and the agency capacity they display, based on the analysis of their life stories. From a care studies perspective, we explore possible lines to (re)signify dependency and autonomy, through the way in which women exercise self-determination, personal affirmation and self-care, and the impacts of these on their subjectivity. We conclude that their determination to exercise self-care is fundamental to the redefinition of their autonomy.

* Correspondencia a / Correspondence to: María Teresa Martín Palomo. Centro de Estudio de las Migraciones y las Relaciones Interculturales (CEMyRI)/Departamento de Geografía, Historia y Humanidades. Universidad de Almería. Cañada de San Urbano, Almería 04120 (España) - tmartinp@ual.es - http://orcid. org/0000-0002-0476-6543.

Cómo citar / How to cite: Venturiello, María Pía; Gómez Bueno, Carmuca; Martín Palomo, María Teresa (2020). Entramados de interdependencias, cuidados y autonomía en situaciones de diversidad funcional. Papeles del CEIC, vol. 2020/2, papel 234, 1-18. (http://dx.doi.org/10.1387/pceic.20940).

Fecha de recepción: julio, 2019 / Fecha aceptación: abril, 2020

ISSN 1695-6494 / (C) 2020 UPV/EHU 
La vulnerabilidad constitutiva de los seres humanos, referida a la susceptibilidad de estos de resultar heridos, pese a ser una condición generalizada, puede verse incrementada en determinados períodos vitales y contextos sociales. Sin embargo, los ideales de la modernidad omiten esta condición inherente al ser humano y arrastran la vulnerabilidad hacia un lugar oscuro y vergonzante (Molinier, 2011), reservado a quienes son etiquetados como dependientes. Y más en particular, a las personas con diversidad funcional (DF), que requieren ayudas para su vida diaria.

Desde los enfoques críticos, la dependencia no es concebida como un atributo de algunas personas, sino como una construcción social originada en las barreras sociales, culturales, arquitectónicas y comunicacionales impuestas a algunas formas de ser y estar en el mundo. El modelo social de la discapacidad sostiene que esta construcción es una forma de opresión (Barnes, 1990), pues no son las personas las "discapacitadas», sino que es el entorno el discapacitante (Gómez Bueno et al., 2011; Martín Palomo, 2010). La discapacidad surge como un término médico utilizado para presentar lo excluido, en tanto reverso de los cuerpos definidos como saludables o capaces (Moscoso, 2016). De este modo, se niega el potencial de los cuerpos y de su diversidad, tras una lógica capacitista, de autonomía y autosuficiencia abstracta, que construye a las personas con diversidad funcional como desviadas y dependientes. El sentido peyorativo asignado al concepto de dependencia es paradójico: por un lado, quienes precisan ayudas para el desenvolvimiento de la vida diaria son estigmatizados por tal condición; por otro lado, ser clasificado como «dependiente» es imprescindible para acceder a las ayudas públicas de cuidados.

La conceptualización del cuidado también es problemática y varía según se atienda a las interrelaciones personales, la naturaleza, los valores o el carácter económico de la relación, el tipo de cuidado o asistencia requeridos, así como al contexto socio-cultural e institucional en el que tiene lugar (Thomas, 1993). A pesar de esta variedad de situaciones, usualmente los sistemas se organizan a partir de la dicotomía cuidador/a-cuidado/a (Martín Palomo et al., 2018), asumiendo que unos proveen y otros reciben atención. Como alternativa a esta mirada, hay muchas formas de ejercer el cuidado y el autocuidado, por ejemplo, mediante la dirección/ control/elección de la ayuda recibida en aquellas actividades que no se pueden realizar individualmente (Morris, 2001; Shakespeare, 2000). De este modo, se ejercita la agencia de quien se supone "dependiente» y se cuestiona la «independencia» de quien brinda los apoyos.

La capacidad de cuidar es culturalmente atribuida a las mujeres como condición natural, lo que las empuja a realizar trabajo no reconocido (Tobio et al., 2010). Sin embargo, cuando las mujeres requieren de apoyos para su vida diaria se sospecha de su capacidad para cuidar a otros (López González, 2008). Las mujeres con DF pueden vivir opresiones que cruzan las desigualdades sexogenéricas con aquellas relacionadas con la estigmatización de sus funcionalidades (Arnau, 2005; Thomas, 2007).

En España, desde comienzos del nuevo milenio se han desarrollado políticas sociales, sanitarias y económicas destinadas a atenuar los efectos negativos del crecimiento de las situaciones de dependencia y a fomentar la autonomía personal al envejecer y/o contar con alguna DF. Las situaciones de crisis (Martín Palomo y Damamme, en prensa; Paperman, 2011), asociadas a los recientes cambios sociodemográficos como el aumento de la esperanza de vida al nacer, el denominado envejecimiento del envejecimiento, la incorporación masiva de 
las mujeres al mercado laboral (Tobío et al., 2010), así como la creciente posibilidad de visibilizar necesidades y demandas por una vida digna, configuran un escenario complejo para dar respuesta a cuidados y asistencias que involucran, en una relación dialéctica, a receptores y/o dadores de apoyos. En este contexto se aprueba la Ley de Promoción de la Autonomía Personal y Atención a las Personas en Situación de Dependencia (Ley 39, 2006) que, siguiendo el ejemplo de los países nórdicos europeos, pretende configurar el cuarto pilar del Estado de Bienestar, junto con los sistemas de salud, educación y pensiones (Cerri, 2015). En la implementación de la ley surgieron algunas dificultades: la crisis económica de 2008 llevó a la disminución de los fondos destinados a su ejecución y algunas de sus propuestas no logran avanzar en la desfamiliarización del cuidado, reproduciendo el trabajo femenino en este ámbito (Martínez Buján, 2014). Respecto a la expansión de derechos universales, no supuso desarrollos notorios, sino que los servicios de cuidado se otorgaron de forma arbitraria y desigual en función de los recursos disponibles, el nivel de renta, el género o la situación de vulnerabilidad social (Artiaga, 2015). En definitiva, en función de la valoración administrativa resultante de cada Comunidad Autónoma.

La Ley 39 (2006) prevé la creación de un Sistema Nacional de Atención a la Dependencia (SNAD), que contempla diversas opciones como: institucionalización (centros de día, residencias), prestación económica para cuidados familiares, servicio de teleasistencia, Asistente Personal (AP) o servicio de ayuda a domicilio (SAD) prestado por profesionales. Las auxiliares del SAD acuden por horas a los hogares de las personas usuarias. Aunque este servicio existía con anterioridad a la creación del SNAD, con la nueva normativa, el servicio amplía su alcance a más población, pero sin dar respuesta a todas las necesidades de cuidado y asistencia cotidianas (Gómez Bueno y Martín Palomo, en prensa). La importancia de este tipo ayudas se refleja, en términos cuantitativos, en la extensión de la población atendida, si bien no hay datos recientes disponibles desagregados para las personas con DF usuarias del servicio'. En términos cualitativos, conllevan cambios sustantivos en la vida cotidiana de las personas adjudicatarias y forman parte del sentido que estas desarrollan sobre el cuidado y la autonomía, ya señalado en otros estudios (Díaz Velázquez, 2010; Muyor y Minguela, 2019; Toboso y Rogero, 2012).

En este artículo se analizan las posibles vías para resignificar la "dependencia», a partir de vivencias encarnadas y situadas. Para este objetivo, el texto presenta un apartado conceptual sobre el cuidado y la interdependencia, seguido por la metodología y los resultados de la indagación cualitativa. Se analizan los relatos de vida de tres mujeres de mediana edad con diversidad funcional, catalogadas oficialmente con "severa" o "gran dependencia», que viven de forma independiente; son mujeres cuya situación de dependencia está asociada a la diversidad funcional y no al proceso de envejecimiento. Se exploran los entramados de interdependencias que participan en la vida diaria de las entrevistadas, así como los cuidados y asistencias que reciben y aportan, que son claves para la constitución de cierta autonomía. Se presentan, asimismo, las percepciones de estas mujeres, consideradas administrativamente

Al 31 de diciembre de 2019, el número de solicitudes de prestación registradas se acercaron a los dos millones (1.894.744), y figuraron como beneficiarias de algún tipo de prestación dentro del sistema 1.115.183 personas. La cantidad de servicios y prestaciones económicas otorgadas ascendió a 1.735 .551 , de las cuales el 69,74\% corresponde a servicios. Tan solo un $0,56 \%$ de las personas que reciben prestación cuentan con AP, un total de 7.837 en todo el país (IMSERSO, 2020). No existen datos desagregados sobre el número de personas con DF que reciben algún tipo de prestación dentro del SNAD, si bien el AP es un tipo de servicio muy demandado por personas con DF (Arnau, Rodríguez-Picavea y Romañach, 2007). 
como "dependientes», acerca del cuidado, la autonomía y la (inter)dependencia en sus experiencias vitales, enfatizando los vínculos, los conflictos, los tiempos y las estrategias de auto cuidado. Finalmente, se presenta la discusión y se concluye que la determinación de sí mismas para ejercer el autocuidado es fundamental para la redefinición de la autonomía.

\section{DEPENDENCIA, INTERDEPENDENCIA Y AUTONOMIIA}

La dependencia se concibe como la necesidad de ayuda de otras personas para el desempeño de determinadas tareas. Esta condición puede ser física, psíquica, emocional, moral y/o económica, e involucra relaciones de dominación y poder. La Ley 39 (2006) clasifica los niveles de dependencia de las personas de acuerdo al tipo de ayudas requeridas para las actividades de la vida diaria: básicas, instrumentales y avanzadas. Mediante estas categorizaciones, el Estado clasifica a los sujetos de sus intervenciones, define qué necesidades deben ser atendidas (Grassi et al., 1994) y diferencia quienes merecen asistencia y cuidado de quienes son excluidos de este derecho. La noción de dependencia ocupa un lugar central en las políticas sociales destinadas a la rehabilitación funcional y la adaptación al medio de las personas con DF.

Sin embargo, determinados paradigmas amplían los sentidos de la dependencia y del cuidado, cuestionando las nociones de autonomía, vulnerabilidad y derechos sociales (Cerri, 2015; Díaz Velázquez, 2010; Martín Palomo, 2010, 2016). Cerri (2015) se refiere a la autonomía relacional que reconoce las interdependencias que permiten el desarrollo efectivo del cuidado, autocuidado y la propia toma de decisiones. En efecto, la interdependencia, red de relaciones que nos caracteriza como seres sociales, queda desdibujada ante nuestras percepciones y sólo la advertimos cuando se vuelve molesta para los parámetros de la cultura en que vivimos (Elias, 1990), por ejemplo, al desafiar la idea de individuo autosuficiente cuando enfermamos y requerimos cuidado.

La autonomía refiere a la capacidad de la persona para obrar según su criterio, o la posibilidad de constituirse en un agente con autogobierno en un contexto social determinado (Kagitcibasi, 2005). Por tanto, concierne a la capacidad de tomar decisiones, y los apoyos para ejercerla serían aquellos que la favorecen y no aquellos que sustituyen la voluntad (Palacios, 2008). El ejercicio de la autonomía se ve constreñido en las poblaciones que ocupan un lugar subordinado en términos de reconocimiento simbólico y económico. Una subjetividad autónoma requiere de la construcción de una intersubjetividad con tales características (Dri, 2002) y con condiciones materiales para desarrollar su autodeterminación. Así, contar con mayor autonomía es disponer de relaciones, en tanto recursos, y de un medio que posibilite su desenvolvimiento. Frente a las barreras sociales, las personas con DF tienen más probabilidades de quedar aisladas, sin más relaciones que las que entablan con sus cohabitantes, más aún, si son mujeres (Moscoso et al., 2014). Las dependencias refuerzan el aislamiento, al limitar las posibilidades de generación y mantenimiento de redes sociales (Venturiello, 2016).

Otro de los riesgos que enfrentan las personas en situación de dependencia lo advierte el Movimiento de Vida Independiente (MVI)2 , cuyos referentes señalan la importancia de prevenir

2 Surgido en el mundo anglosajón hacia 1970 y formado por personas con DF que defienden su derecho a elegir y decidir dónde vivir, qué cosas hacer y cómo, así como libertad para participar en la comunidad (Evans, 2001). 
diferentes formas de violencias hacia estas personas por parte de cuidadores/as e instituciones (Arnau et al., 2007; Evans, 2001). Por tanto, atender a la autonomía de las personas con DF requiere establecer mecanismos de asistencia para que los sujetos no sean oprimidos ni en sus casas ni en instituciones (Arnau et al., 2007; Morris, 2001). Según este colectivo, ciertas prácticas de cuidado biomédico pueden atentar contra la autonomía y el derecho de quienes asisten, pues al tiempo que ofrece curas, la biomedicina impone formas de dominación que convierten a los "pacientes» en cuerpos/objetos de intervención, desprovistos de subjetividad, voluntad, contexto e historia (Conrad, 1982). En el caso de las mujeres con DF, además, se ha constatado su mayor exposición a sufrir violencia sexual y de género en las instituciones, como también en el ámbito familiar (Moscoso, Martín-Palomo y Muñoz Terrón, 2014).

Como alternativa a estas situaciones, la figura del AP se asocia al modelo de autonomía personal y propone reformular las relaciones, favoreciendo el establecimiento de vínculos de mayor reciprocidad y menor afectividad y violencia (Artiaga, 2015). Ello no implica desterrar ni las contradicciones ni la violencia que puede suscitarse en el cuidado, puesto que, en tanto relación humana, este genera vínculos afectivos (Martín Palomo, 2016). Es más, las violencias serían susceptibles de ser ejercidas también en sentido inverso ya que, en ocasiones, serían las personas cuidadas las que violenten a las personas con las que interactúan en su día a día, sean colegas, familiares o profesionales. Esta afirmación de las capacidades agenciales y de toma de decisiones puede implicar el ejercicio del poder sobre cuidadores/ asistentes, y abre la cuestión de la vulnerabilidad en las condiciones de contratación de cuidadores que, por falta de espacio, no se aborda en este artículo. Sin embargo, el modo en que las mujeres con DF, que viven donde han elegido vivir y con quien quieren hacerlo, pueden ejercer la autodeterminación en la gestión de su cuidado se muestra como un territorio aún poco explorado. A continuación, presentaremos cómo gestionan las relaciones en las que participan.

\section{MATERIALES Y MÉTODOS}

Este trabajo forma parte de un proyecto de investigación más amplio 3 cuyo objetivo general es analizar las prácticas y los significados atribuidos al cuidado, la autonomía, la autodeterminación, la dependencia y las interdependencias de las personas insertas en entramados de cuidados en Andalucía y Madrid. Su primer objetivo es examinar los modos en que organizan su vida diaria las mujeres con diversidad funcional severa que cuentan con servicios de ayuda a domicilio (SAD) públicos ( $y$, en ocasiones, también privados), así como las relaciones de interdependencia que intervienen en ello y la capacidad de agencia que desarrollan. El segundo objetivo explora posibles líneas de resignificación de la dependencia y la autonomía a partir del modo en que estas ejercen la auto-determinación, la autonomía y el auto-cuidado. El abordaje es de tipo cualitativo, con una muestra intencional, a partir de relatos de vida como herramienta de producción de información (Bertaux, 2005).

3 Artículo escrito en el marco de dos proyectos de investigación: a) El portal del familiar-GESAD Family financiado por el Ministerio de Economía, Industria y Competitividad. Ref. AEI-010500-2017-225. Universidad de Granada (2017-2018); b) Beca postdoctoral de Asociación Universitaria Iberoamericana de Postgrado otorgada a María Pía Venturiello, para realizar una estancia de investigación sobre cuidados, género y población en situación de dependencia en la Universidad de Granada (2018). 
Se presentan aquí los resultados referidos a tres mujeres con DF, administrativamente clasificadas con "severa o gran dependencia», y con capacidad de gestionar su cotidianeidad. Estos fueron los principales criterios de selección de las informantes, pues son mujeres con DF que viven independientes de sus familias y que son activas al organizar su cuidado. Además, se atendió a diferentes tipos de DF, el tiempo vivido en situación de dependencia y su militancia. Esta diversidad provee de variaciones discursivas sobre el modo de ejercitar y dar sentido a la autonomía y la dependencia. En cuanto a sus características sociodemográficas, dos de ellas cuentan con estudios superiores, trabajan y militan por las personas con DF, con una desarrollada conciencia de los derechos del colectivo; y la tercera es jubilada, tiene estudios secundarios y sus ingresos proceden de una pensión por DF, trabajo y jubilación. Reciben ayudas del SAD y, si pueden, contratan cuidadoras/es remunerados adicionales. Todas ellas viven habitualmente solas, no tienen hijos, y en algunos períodos se encuentran en convivencia con su pareja o algún familiar. Como resguardo ético se utilizaron seudónimos para preservar el anonimato de las entrevistadas. Dibujamos un pequeño perfil de cada una de esas mujeres:

- Marcela, 44 años, vive en una residencia universitaria en el medio urbano y trabaja como investigadora académica. Presenta una DF congénita. Su familia reside en otra región. Tras varios años internada, cuenta con asistencia personal completa que paga de manera privada; sus AP significan un importante avance a favor de su autonomía, pues adoptan la filosofía del Movimiento Vida Independiente (MVI), en el que Marcela milita.

- Laura, 34 años, vive intermitentemente sola y con pareja, en el medio urbano. Es estudiante universitaria. También conoce el MVI y se adhiere a su enfoque sobre los derechos del colectivo. A los 26 años sufrió un accidente y adquirió una tetraplejia para cuya rehabilitación pasó internada un año. Actualmente complementa el cuidado del SAD con el prestado por cuidadoras contratadas a través de una asociación y de forma privada. Para lograr la asignación de más horas del SAD reclamó con firmeza la revisión de su grado de dependencia.

- Celeste, 45 años, vive intermitentemente sola y con pareja, en el medio rural. Jubilada recientemente. Está diagnosticada con enanismo y por deterioros en su salud y a consecuencia de una caída, ve incrementadas sus necesidades de atención en la vida cotidiana. Trabajó durante años en el Ayuntamiento de su municipio como auxiliar administrativo. Hasta que recibió la ayuda del SAD fueron su hermana y padre quienes atendieron sus necesidades de cuidado.

El análisis cualitativo de los relatos de las entrevistadas se organizó a partir de dimensiones conceptuales, algunas de las cuales se establecieron antes del trabajo de campo y otras emergieron en relecturas posteriores, y se agrupan en: (a) tareas del cuidado: aspectos materiales, morales y emocionales; (b) el vínculo con cuidadores/as y asistentes: expresiones sobre la dependencia y la autonomía, estrategias de (de)construcción de la dependencia, negociación e interacción entre las personas implicadas en el cuidado, los límites del cuidado; y (c) participación de familiares e instituciones en el cuidado: las paradojas de los sistemas familiaristas y burocráticos, la autonomía de las personas involucradas en esos vínculos y las relaciones e interdependencias con los espacios institucionales proveedores de cuidado.

Entre las limitaciones del estudio se destaca el reducido número de informantes y la poca representatividad del colectivo en términos de nivel educativo. No obstante, esos rasgos cons- 
tituirian también una fortaleza pues lo que se busca a través de estas mujeres con estudios, empoderadas y con gran capacidad de agencia es, precisamente, explorar las condiciones de interdependencias que han generado espacio para la autonomía, la militancia y la lucha. Aunque quizás minoritarias, permiten construir un marco y unos sentidos de la autonomía fuera de sus concepciones hegemónicas.

\section{EL CUIDADO Y LA DIVERSIDAD FUNCIONAL EN LA VIDA COTIDIANA: HACIA LA AUTONOMİA Y LA INTERDEPENDENCIA}

\subsection{Vínculos e interdependencias: las tramas del cuidado}

El cuidado incluye todas aquellas acciones realizadas para el mantenimiento de la vida cotidiana (Fisher y Tronto, 1990) que, usualmente, resultan invisibles (Molinier, 2011). La necesidad de cuidado alcanza a todas las personas y no es un requerimiento exclusivo de quienes se encuentran en situación de dependencia severa, aunque estas puedan precisar de apoyos específicos. Las informantes requieren de ayuda para levantarse, vestirse, comer y salir de sus hogares hacia las actividades laborales, educativas o recreativas. Tales necesidades evidencian el entramado de relaciones que sostienen su vida.

Las entrevistadas cuentan con redes familiares y comunitarias para su vida diaria. Asimismo, combinan las ayudas para tareas específicas que brindan las cuidadoras del SAD con las prestadas por personal contratado. Laura participa, mediante el pago de una cuota mensual, de una asociación de personas con DF que le provee de cuidadoras por un coste menor al del mercado. Marcela, cuenta con AP formados/as en las oficinas del MVI y Celeste solo cuenta con la auxiliar del SAD enviada por el ayuntamiento. Así, estas mujeres gestionan su cuidado a través de los servicios proporcionados por la administración local o autonómica, asociaciones o contratos privados. De acuerdo con sus relatos, las cuidadoras del SAD no alcanzan a atender sus necesidades básicas ni instrumentales, y menos aquellas de desarrollo personal; para alcanzar sus metas requieren recursos propios.

Los contornos del trabajo del cuidado no siempre son claros y están en permanente negociación. Cuidar supone una dimensión material que implica unas cualificaciones tácitas (adquiridas a través de la socialización familiar y no reconocidas ni valoradas por el mercado), y otras explícitas, adquiridas a través de la educación formal y la experiencia profesional (Gómez Bueno, 2000). Las cuidadoras o AP de las entrevistadas se ocupan diariamente de brindar apoyo en las tareas básicas: levantarse, comer, asearse, salir y, en ocasiones, también del trabajo doméstico. En el caso de Marcela, las actividades desarrollas por el AP incluyen el acompañamiento en las actividades básicas, laborales y recreativas: le asisten para leer, para ir a una conferencia o al cine. Para Laura, con problemas severos de movilidad, el aporte más destacable de sus cuidadoras se desarrolla dentro del hogar. También Celeste describe la asistencia que recibe centrada en su rutina de cuidado personal e instrumental diario, de forma pasiva cuando habla de su cuidado corporal en el entorno doméstico, y de forma activa cuando se refiere a salir fuera de su hogar:

Viene por las mañanas, estoy en la cama, me levanta, me pone en el baño, hago mis necesidades y luego me pone en la bañera, en la ducha, y me baña, me asea, 
me viste, me ayuda a vestirme y demás. Luego, una vez vestida y una vez arreglada pues se pone ella con las tareas de la casa (...) Y ya me hace las tareas y luego ya termina y luego paseamos, salimos a comprar. (Celeste)

El papel que, según nuestras informantes, desempeñan cuidadoras/es profesionales y familiares en sus entramados de interdependencias es paradójico: por un lado, rechazan las redes familiares por el riesgo de sobreprotección, paternalismo e infantilización que les atribuyen, apostando por su independencia. Por el otro, las redes de cuidadores profesionales están sometidas a lógicas burocráticas ( $y / 0$ mercantilistas) que posibilitan cierta autonomía, pero que emplean prácticas de despersonalización y deshumanización, a lo que también se oponen.

No obstante, las familias suelen ofrecer apoyos ante urgencias y situaciones límite. Ellas describen a sus familiares cercanos como ajenos a su cotidianeidad y ocupados con sus tareas y problemas personales. Aunque su familia viva en el mismo pueblo y mantengan estrechos vínculos afectivos, esta no configura su red básica de cuidado:

No tengo madre, tengo a mi padre, está muy mayor... y es un hombre, que no. Pero me llevo bien con él y todo. Y tengo una hermana, ya está [resopla] pero ella tiene dos hijos, trabaja y la veo poco (...). (Celeste)

En tanto que quieren mantener su independencia y ejercer su capacidad de agencia, declaran no desear ser cuidadas por sus familiares o parejas:

Mi pareja no debería ser en ningún momento mi AP, ni mi hijo, ni mi madre, no creo que deban ser mis asistentes personales, porque para poder ejercerlo como rol de hija o de madre o de compañera sentimental creo que tengo que tener cierta independencia. (Marcela)

Esta lógica prioriza la ayuda extra-familiar remunerada como estrategia para resguardar su autonomía, huir de la dependencia emocional y del sentimentalismo y evitar preocupaciones y cargas a sus allegados. Frente a la proximidad afectiva y el lazo emocional que regulan las interacciones familiares optarán por la lógica mercantilista que posibilita la autodeterminación, la distancia emocional y el empoderamiento: contratar, seleccionar, exigir y pagar.

El cuidado puede ser proporcionado sin mayores conflictos por un AP varón y desafiar así el orden patriarcal ${ }^{4}$, pero no por un familiar. Este componente de la red se activa solo ante un fallo grave en la provisión de asistencia. Laura describe como, en una ocasión durante dos días, las cuidadoras fueron enviadas por el ayuntamiento en un horario equivocado, en el cual ella no contaba con su pareja o vecinos para que abrieran la puerta $y$, antes que llamar a sus padres, prefirió esperar sin asistencia en su casa:

Llamaba a mis padres que tenían que venir desde mi pueblo y ellos no tienen por qué, porque es un derecho que me pertenece. Yo llamaba por teléfono, llamé una vez antes de que tuvieran la autorización de llave, mi hora es a las once y venían a las ocho y yo a las ocho no tenía a nadie que le abriese la puerta, mis vecinos a esa hora están durmiendo (...) Es que claro, porque yo no quiero llamar a mis padres para preocuparles, para decirles: «Mamá, es que llevo dos días aquí sin comer ni beber». (Laura)

4 Es el caso de Marcela, las demás cuidadoras son mujeres en consonancia con la feminización del sector. 
En los entramados de interdependencias, cada componente ocupa el rol asignado por las entrevistadas. Así, a los miembros de la red familiar les asignan un rol de refuerzo ante las contingencias, que se complementa con las redes de vecindad para casos muy concretos, como abrir la puerta a una cuidadora. Las redes de amistad participan en actividades puntuales y festivas como arreglarse juntas para salir. Las parejas afectivas, por su parte, tienen asignados roles diferentes según la informante. Mientras que la de Laura puede compartir parte de la atención cotidiana (aunque prefiere que lo haga el AP), la pareja discontinua de Celeste, durante el período de convivencia, se implica en actividades domésticas y de cuidado personal. El relato muestra esta participación como algo espontáneo, acotado a ciertos períodos, sin ocasionar disrupciones dentro de la pareja:

Estoy viviendo con una persona, pero ya se tiene que ir, es de Córdoba y es (...) pues él me hace de comer (...) Y la muchacha pues lo que quiera, si me tiene que hacer de comer, me hace. Pero por ahora, como está él pues ya dice: «no voy a estar aquí sin hacer nada». Y hace la comida y demás, pero que ella también me hace. (Celeste)

Así, el cuidado conlleva una división del trabajo (Molinier y Legarreta, 2016) que responde a condiciones organizativas y económicas, a las ofertas institucionales y las pautas morales que comprometen a las personas de las redes de interdependencia (familiares, amistades, profesionales, parejas) en una tensión entre la percepción y definición de las necesidades, el deber de atención, los afectos y el respeto a la autonomía y la capacidad de agencia (dejar decidir y hacer).

\subsection{Rotación de cuidadoras/es: descuidos burocráticos y conflictos}

En las situaciones de "severa o gran dependencia» se desafían las ficciones de autonomía plena y se advierten las interdependencias que nos constituyen. El vínculo del cuidado entre adultos autónomos supone construir pactos, aprendizajes sobre cómo vincularse y conocimiento mutuo para diseñar un mundo común. Requiere, por tanto, cierta estabilidad de la red. Por ello, la dinámica de rotación de cuidadoras/es que impone el SAD genera dificultades para construir este código común necesario para su bienestar. Celeste, con nuevas necesidades, identifica lo peor de los cambios como:

Adaptar... a que me... a ver otra chica nueva, que vea como me... todo lo nuevo, otra vez de nuevo.

Laura, con años requiriendo asistencia para su vida cotidiana, reclamó la continuidad de la cuidadora. Al utilizar sonda, precisa asistentes con cualificaciones específicas. Las rotaciones, falta de formación e inexperiencia de las cuidadoras pusieron en riesgo su salud en varias ocasiones. Tras extenuantes jornadas reclamando, logró que la incluyeran en «casos especiales»:

Casos especiales es que tú tienes una misma cuidadora fija todos los días, quitando los fines de semana. Porque claro, yo tengo sonda y tengo que enseñarles y son cosas delicadas, que más de una vez me he visto en el hospital porque me han mandado auxiliares de limpieza y ellas [no tienen los conocimientos]. (Laura) 
El trabajo de preocuparse y ocuparse del otro (Tronto, 1993) se ve entorpecido por la dinámica despersonalizada de la asignación burocrática de asistentes. No obstante, prefieren la respuesta flexible de cuidadoras/es profesionales antes que el cuidado de familiares, también elástico ante las eventualidades, al tiempo que se opone a la lógica burocrática que fundamenta el proceso de solicitud, valoración, respuesta. Desde la asignación del grado de dependencia hasta la adjudicación de las horas/dias (siempre escasas en opinión de las entrevistadas), los horarios y las tareas a cubrir son procesos cuestionados o rechazados. Las entrevistadas perciben una abstracción y despersonalización de su realidad y sus necesidades, aunque también reconocen las dificultades que supone dar respuesta satisfactoria a las mismas, así como la precariedad laboral de las cuidadoras:

\begin{abstract}
Yo tengo una tetraplejia a nivel C5-C6. Tengo un 90\% de discapacidad. A mí cuando me valoraron estaba estudiando derecho y yo sabía que por ley un tercer grado, antes un $90 \%$ era un tercer grado, ni siquiera te debería de valorar lo que es el grado, sino el nivel. Entonces me dieron grado 2, nivel 2 y me han dado sólo 48 horas. ¿Entonces qué pasa? Que yo vivo sola, tengo que pagar a mujeres que me limpien porque la de la dependencia viene media hora por las noches nada más y en esa media hora tengo que aprovechar para acostarme y cenar. (Laura)
\end{abstract}

Laura muestra algunas de las dificultades que desafían al programa del SAD como la valoración de las necesidades, la asignación de servicios y la contratación de personal cualificado. Refleja la ambigüedad en la definición de las tareas y cualificaciones necesarias para el cuidado. Las fronteras entre el trabajo doméstico y el trabajo de cuidados/AP son objeto de constantes luchas y reivindicaciones. En la formación del AP se prioriza la mirada respetuosa sobre la vida independiente. En contraste con las demás, Marcela tiene una visión más positiva, posiblemente por contar con AP con quienes comparte una filosofía de vida y a los cuales ha entrevistado y seleccionado ella misma. Si bien implica un esfuerzo tratar con muchas personas, también lo describe como una oportunidad que contrasta con otras experiencias vivenciadas durante internamientos, donde su parecer estaba relegado.

\title{
4.3. Los tiempos del cuidado: desencuentros y reencuentros
}

El tiempo es una de las dimensiones sensibles del cuidado. La falta de coordinación de los miembros de la red puede conllevar una situación muy crítica para la persona en situación de dependencia y una exigencia incluso insalubre para la empleada que cuida. Mientras las personas con severa o gran dependencia esperan tiempo de más, las auxiliares del SAD corren para llegar a tiempo y se producen desencuentros evitables, originados en la escasez de recursos institucionales. La prisa con la que deben cumplir ciertas tareas y la espera obligada de la persona que recibe el cuidado forman parte de una dinámica en la que se ven limitadas ambas partes. Laura describe cómo debe adaptarse a los breves tiempos en los que cuenta con cuidadoras para atender sus necesidades fisiológicas, y nuevamente da cuenta de forma pasiva de las atenciones recibidas, quedando en cierto modo enajenada su agencia:

-Viene y me pone en el sofá o me pone a hacer pipí y se va. Y ya está. O me pone la comida, esa media hora la tengo para todo eso.

-Y luego la otra media hora por la noche. 
-Para acostarme. Hacer pipí y acostarme.

-Entonces te tienes que acostar a la misma hora siempre

-A las nueve, claro. (...) Nos tenemos que adaptar. (Laura)

En este reparto de horarios, con el que no se responde de manera satisfactoria a las necesidades de las personas involucradas (cuidadas y cuidadoras), se genera un descuido institucional. Tras sortear las confusiones de horarios se produce un nuevo encuentro. En esa interacción, una vez más se actualizan las dimensiones materiales, simbólicas y emocionales del cuidado desde las distintas partes y se construyen acuerdos más o menos tácitos. Ante descuidos y retrasos, las entrevistadas contemplan, como empleadoras, la situación de estas trabajadoras. Laura y Celeste coinciden en que los retrasos no son atribuibles a las cuidadoras, por lo que no van a ejercer su poder contra ellas:

A mí una cuidadora me viene tarde, porque la han puesto 20.000 servicios a la pobre, y viene de autobús en autobús casi sin aliento, yo no la voy a regañar, por supuesto, porque no es culpa suya. (Laura)

La ausencia o retraso de la cuidadora/asistente supone un alto coste para ellas, aun cuando lo comprendan. Marcela, reconoce los derechos de descanso de los AP, aunque ello implique alterar sus rutinas y posponer actividades básicas, y Celeste lamenta las restricciones presupuestarias que tienen claras consecuencias sobre el cuidado que recibe:

Como es un presupuesto mínimo lo que dan pues el día que ella no viene, como hoy, que se ha pedido el día para ir a ver a su hija a la función del colegio, pues yo... pues no me he podido levantar hasta medio día a ducharme. (Celeste)

La relación de dependencia e intercambio emocional se da en el marco de vínculos entre adultos responsables de si mismos, en la que se comparten valores relativos al modo de cuidar. Así, aunque requieran ayuda y cuidado, no dejan de ser personas autónomas con la posibilidad de construir y romper vínculos. Estas dimensiones afectiva, moral y emocional del cuidado son rescatadas por las entrevistadas. Recibir de sus cuidadores/as una actitud comprensiva produce dos efectos: (1) el respeto de las decisiones de quien es cuidado, enfatizado por Marcela, pues supone el reconocimiento de su autonomía; y (2) el trato afectuoso y cálido, valorado así por Laura y Celeste:

«Hola, buenos días [tono dulce]. Venga, espabílate mientras te abro la persiana y te preparo la ducha». Pues son unas formas bonitas, humanas de decir... de trabajar con gente como nosotros. (Laura)

El estar sola y que venga una persona y que entre por la puerta, eso ya es mucho, que te digan buenos días y... eso para mí es mucho. Ya estar un ratico hablando y ya pues que haga la limpieza. Pero el entrar alguien por la puerta (...) Si, eso es, el trato. Tener un diálogo con la persona, contarle tus cosillas "¿Qué te pasa hoy?" "Pues hoy estoy muy triste». Tener un desahogo. (Celeste)

Aqui se evidencia la dimensión emocional del trabajo de cuidado puesta en juego, al saber respetar y escuchar (Hochschild, 1983) o al saber mantener la distancia adecuada. Además, destacan la importancia de construir un vínculo de confianza:

Yo creo que ser AP es una de las profesiones más difíciles que pueden existir en el mundo hoy en día, porque tienes que ser paciente, fiel, yo debo poder confiar en ti. 
Y fiel en todos los sentidos, tienes que ser discreto o discreta, porque tú quizás lo sepas todo de mí, tampoco es cuestión de que vayas contándolo por ahí. (Marcela)

Fidelidad, respeto, confianza son cualificaciones requeridas y valoradas en cualquier relación y también en la que se establece en el cuidado. Tal vez aquí, ante relaciones marcadamente asimétricas, sea el respeto por las decisiones de la persona en situación más vulnerable lo más valorado por las informantes (Morris, 2001; Shakespeare, 2000).

\subsection{Resignificando la dependencia: autodeterminación, reconocimiento y autocuidado}

Una de las críticas a la Ley 39 (2006) es que no distingue entre dependencia (limitaciones para actividades motrices o cognitivas) y la posibilidad de ejercicio de la autonomía (Cerri, 2015): la dependencia parece poner en cuestión la autonomía. De ahí que Moliner (2011) sostenga que a veces cuidar es dejar hacer al otro o dejar que este decida. Si la autonomía se concibe de acuerdo con parámetros abstractos que omiten las interdependencias personales, cuando la necesidad de ayudas y el trabajo cooperativo inherente a la condición humana se vuelven evidentes ante determinadas situaciones de salud, se produce una oportunidad de redefinición de su sentido dominante.

Al respecto, Marcela enfatiza el rol de los AP en el entramado de interdependencias que configura sus condiciones de vida y su autonomía:

Mis asistentes personales trabajan en algo muy complejo que es mi libertad, yo soy libre si y solo si tengo AP. Y hoy en día yo digo que solo puedo ser persona si y solo si tengo AP. Yo quiero comer como yo quiera, quiero comer a mi manera. No me basta con que me dé de comer cualquiera, no me basta que me limpie el culete cualquiera. (Marcela)

Ciertas ambigüedades y contradicciones entre los discursos de las informantes remiten a la activación de dos marcos semánticos: (1) de desaprobación y extrañamiento del yo, tiempo pasivo de la cita anterior: "que me dé de comer cualquiera»; "que me limpie»; y, (2) de afirmación personal y expresión de autonomía, en primera persona: "me levanto", "me baño»..., como en el siguiente verbatim, en el que Laura cuenta que su situación de «dependencia severa» no supone una restricción a su autonomía:

A esa hora me levanto y me pongo a desayunar y luego me baño, me pinto, me arreglo un poco y a las nueve menos diez o a las diez... No, perdón, sobre las diez o las doce, dependiendo del día, del horario que tenga de clase, pues ya estoy abajo esperándoles. (Laura)

Sin embargo, estas formas de afirmación personal son interpretadas erróneamente desde las instancias de evaluación burocráticas, para quienes dependencia y autonomía se consideran categorias antagónicas. Laura salió perjudicada del proceso de valoración de su grado de dependencia precisamente por el campo semántico empleado:

Ahora te valoran, te hacen preguntas. Claro, entonces, si yo decía: «¿Necesitas ayuda para la ducha?». Y digo: "Yo me ducho sola, sí, pero necesito ayuda para las 
piernas o para el pelo o cosas así». Te cuentan un +1 , te cuenta como que lo haces tú sola. (Laura)

Las entrevistadas experimentan esta oposición en las formas de sentir su autonomía y en las clasificaciones externas. Ellas desarrollan una lucha por su autonomía y un trabajo de reafirmación que contribuyen a activar el marco semántico de afirmación personal y empoderamiento. Sin embargo, la lógica burocrática, insensible a esos laboriosos avances, actúa en contra del logro de la autonomía personal, contribuyendo a que su ejercicio no esté al alcance de todos los grupos sociales. La informante con menos estudios y sin capital militante se mueve en el marco semántico del extrañamiento del yo, sin lograr activar el marco de la auto-afirmación personal.

Marcela sostiene que desde que tiene AP "por primera vez me cuido a mí misma», dejando atrás las experiencias de internamiento en uhospitales o residencias, en los que los horarios para comer, asearse y otras necesidades estaban reglados según un modo estandarizado de producción de la atención. Este tipo de situaciones se reproducen de algún modo en los hogares cuando nuestras informantes tienen que adaptar sus tiempos de descanso y actividad a los horarios de las cuidadoras asignadas por el SAD, como mencionaba Celeste. Laura y Marcela, tras pasar por experiencias de internamiento, insisten en sus aspectos deshumanizantes:

Cuando a uno le apetece hacer pipí se hace pipí, y si hay cuidadoras aquí toda la mañana trabajando tiene que haber cuidadoras para que le ayuden al baño. No, que a las doce toca hacer pipí. No, no ¿Por qué? Entonces yo me hago caca en el cuarto, te la cambian y te dejan el pañal ahí todo el día. Eso es antihigiénico. Ellas decian que no era su trabajo. (Laura)

Estas cosas que parecen habituales para el resto de las personas, para nosotros, las personas con DF, cuando necesitamos apoyos eso parece que ya no tiene importancia, y... Te ducharán cuando puedan, te darán agua cuando puedan, te pondrán a hacer un pipí cuando puedan... Ya no eres tú. (Marcela)

No obstante, los modos de ejercer la autonomía se aprenden con la práctica. La misma Laura reconoce la importancia del centro de rehabilitación donde se recuperó para desarrollar una actitud independiente. Alli se aplica el principio moral de "valerse por sí solo» desarrollado en ciertos campos de la DF como estrategia para afrontar un mundo que expulsa a las personas concebidas como tales (Ferrante, 2014), incluidas aquellas prácticas e instituciones que niegan sistemáticamente su autonomía:

Yo he estado en el [centro de rehabilitación] de San Fernando. Allí tienes que ir para formarte tanto intelectual como físicamente. Entonces, si tú no quieres ir al gimnasio, a ti te echan. (...) Había un chaval que estaba allí siempre con la madre, cuando llegó al centro, pues le acabaron diciendo: "O se va usted o se van los dos. Porque este centro es para independizarse hasta donde pueda. Que él puede hacérselo todo después y magnífico, pues bien; que él no puede hacérselo todo y hasta donde llega ha llegado, pero por lo menos saber vestirse, desvestirse, pasarse a un coche y se ha llevado su carnet de conducir y todo, pues mejorn. (Laura)

Una parte fundamental del cuidado supone dejar lugar para el autocuidado como una manifestación de autonomía. El ejercicio del autocuidado se encuentra en los pequeños actos, por ejemplo, el decidir sobre el momento para una ducha o una comida; sobre qué se quiere co- 
mer, con qué condimentos, cuándo, cómo y con quién. Esto no excluye la necesidad de ayudas. Al contrario, el entramado de interdependencias es el que posibilita el autocuidado: si el otro me ayuda respetando la propia decisión, el/la asistente/cuidador/a es una posibilidad para ejecutar, y no obturar, el autocuidado. En este sentido, la educación moral ligada al cuidado y al autocuidado activa el marco de construcción del sentido desde la afirmación personal. Marcela lo explica así:

Yo, desde que tengo asistencia personal, yo me cuido (...). Yo tomo la decisión... pues, de cuando quiero comer, qué como, si como bien, si como mal, si me quiero bañar todos los días, la higiene, todo eso no lo determina un profesional, lo he determinado yo. Yo soy la que dice: «pues hoy me apetece, pues hoy no me apetece». (Marcela)

Mientras que Laura, desde su condición de dependencia severa adquirida, toma conciencia de que: «ahora no estoy sola». Cuidarse y ejercer la propia autonomía supone para ella tomar conciencia de los entramados en que está inmersa, y gestionar las redes de interdependencia, los intercambios que implican la negociación de las esperas (Allué, 2005), los acuerdos y el respeto mutuo:

Nosotros al principio nos acostumbramos a que: «iven!» y tiene que ser ya, porque
tú antes te servías un vaso de agua cuando te daba la gana, lo que no te enteras
es que ahora ya no estás tú sola, tienes a otra persona que te lo está haciendo con
todo el cariño del mundo y la estás tratando muy mal, no estás pensando en que...
(Laura)

Celeste relata cómo fue pasando de una lógica de construcción del sentido de la autonomía a otra; de pensar que comenzar a tener asistencia profesional era una pérdida de independencia a interpretarlo como una ganancia:

Trabajaba en el Ayuntamiento, pues ya me informé bien y me dijeron la ayuda que podía tener con la auxiliar del SAD. Porque yo era reacia a eso porque yo me veía bien como para... Pero luego me he alegrado mucho (...) como he sido muy de salir, yo me veía en mi trabajo y todo. Y digo: "¿para qué la necesito?». Pero ya es que fue todo empeorando, ya me jubilé por la enfermedad, porque iba a peor y pasé por el tribunal y me dieron la gran dependencia, la gran... (Celeste)

La noción de autonomía y, derivada de esta, la del autocuidado, se van construyendo y transformando a lo largo del proceso cotidiano de requerir ayudas, recibirlas y entablar rutinas, procesos y acuerdos con quienes forman parte de sus vínculos.

\section{CONCLUSIONES}

El presente artículo analiza los modos en que organizan su vida diaria mujeres con diversidad funcional que cuentan con el Servicio de Ayuda a Domicilio (SAD). En los relatos se descubren sus caminos para resignificar la "dependencia», el modo en que ejercen la auto-determinación, la afirmación personal y el auto-cuidado. Las informantes nos ilustran sobre cómo viven su autonomía, a pesar de ser burocráticamente clasificadas con «severa» o "gran dependencia». 
La reconstrucción de los intercambios materiales y simbólicos que se dan en entramados de interdependencias de los que forman parte - familia, cuidadores/asistentes, instituciones-, ha permitido revisar el contexto en el que despliegan su capacidad de agencia. La red familiar es la principal fuente de recursos ante las situaciones de dependencia en las culturas mediterráneas. Sin embargo, la distancia afectiva para establecer códigos y negociaciones entre quien cuida y quien es cuidado se desdibuja entre familiares. Por ello, las entrevistadas relegan sus colaboraciones a situaciones puntuales y excepcionales. Sus aspiraciones vitales de prescindir de las atenciones cotidianas familiares, se contraponen a la estructura social y a las políticas familiaristas que existen en España. Aquí, la familia se asume elástica, flexible, siempre "preparada" para dar respuesta a cualquier necesidad sobrevenida. Frente a la proximidad afectiva y emocional que regula la interacción familiar, optan por el cuidado mercantilizado y/o burocratizado, distante y especializado en el respeto a los derechos individuales, lo que incluye prácticas relacionales que preservan y fomentan la autonomía: elegir, decidir, organizar aspectos concretos de su vida.

Como alternativa superadora a las atenciones de personas afectivamente muy próximas, las entrevistadas cuentan con diferentes tipos de ayudas extra-familiares: auxiliares del SAD, cuidadoras contratadas en el mercado mediante empresas, asociaciones o AP a través del MVI. En el vínculo que establecen con sus cuidadoras/asistentes valoran el respeto mutuo, el buen trato y la confianza. Asimismo, destacan el tiempo que les lleva construir esa relación con las o los profesionales que atienden sus necesidades para la vida cotidiana, aspecto que debe ser contemplado por las gestiones públicas que garantizan esta provisión. El vínculo de cuidado supone riesgos de violencia que las entrevistadas expresan haber vivido, especialmente, en las instituciones de internamiento. Sin embargo, las interrelaciones actuales, aunque presentan ciertos desajustes, expresan la toma de conciencia y la disposición al respeto recíproco de los derechos y la autonomía de cada cual. Como muestra de ello, las entrevistadas refieren las condiciones de precariedad laboral en que ejercen su trabajo sus asistentes y cuidadoras, entendiendo sus demoras u otras dificultades. El escaso reconocimiento del cuidado de personas en situación de dependencia, retroalimenta la situación de vulnerabilidad del colectivo.

Asimismo, las informantes enfrentan los efectos de la imposición de una lógica burocrática que contrapone "dependencia» y «autonomía». Se critica la Ley 39 (2006) por no distinguir entre "dependencia" y "posibilidad de ejercicio de la autonomía" (Cerri, 2015), lo que supone una forma de violencia: la negación de la autonomía de la persona cuidada. Estas categorías burocráticas producen realidad. A través de su imposición se restringe la idea de autonomía puesto que, por definición administrativa, según la Ley 39 (2006), si eres una persona autónoma no necesitas el SAD. Por ello, reclamar simultáneamente ayudas en la vida cotidiana y aspirar a la autonomía, se percibe como contradictorio, y supone una lucha por el reconocimiento del entramado de interdependencias y de la capacidad de autodeterminación de quien es considerado "dependiente» como un logro de afirmación personal. Las estrategias que desarrollan las sociedades para brindar apoyo y cuidado participan incrementando o deteriorando la autonomía de estas personas en ámbitos sensibles de la vida diaria.

Las entrevistadas que cuentan con una formación superior activan un marco semántico de afirmación personal y empoderamiento, que denota la ejecución por sí mismas de tareas como: comer, asearse, ir al baño y vestirse, aunque otras personas estén implicadas en su realización. Así, el sentido, ejercicio y contenido de su autonomía se construye y practica en diferentes entramados de interdependencias. Lugar preferente ocupan las relaciones inter- 
personales con cuidadores/asistentes remunerados/as, en interacción con las redes de apoyo familiares y de amistad, la universidad, la militancia y el asociacionismo. En otro campo semántico se mueve la entrevistada con menos estudios, con una red de apoyos más limitada y sin capital militante, al activar un marco semántico del extrañamiento del yo: "me pone en la bañera», "me baña», "me asea», opuesto a un marco de auto-afirmación personal.

En la asistencia a personas adultas con DF, las condiciones de posibilidad de un ejercicio respetuoso de los derechos humanos (Morris, 2001) y de la autonomía están marcadas por prácticas burocráticas contradictorias, resueltas con cuidadoras/asistentes con diferentes cualificaciones y motivaciones. A su vez, implica disputas políticas por el sentido de la dependencia y el reconocimiento del cuidado, que respete el autocuidado como parte de la autonomía, y que evidencie todas las formas de dependencias interpersonales y sociales, y no solo algunas (Cerri, 2015). El incremento demográfico de la población en situación de dependencia, constituye una oportunidad para construir nuevos modos de organización que reconozcan nuestra vulnerabilidad constitutiva y la autodeterminación como partes imprescindibles de la complejidad del cuidado.

\section{BIBLIOGRAFÍA}

Arnau, M.S. (2005). La cara oculta de la violencia: la Violencia de Género contra la(s) Mujer(es) con disCapacidad(es). GIAT Discapacidad-Fundación Isonomía Igualdad Oportunidades, Universidad Jaume I. Disponible en: https://sid.usal.es/idocs/F8/FDO9078/estudiocara-oculta-violencia.pdf

Arnau, M.S., Rodríguez-Picavea, A., y Romañach, J. (2007). Asistencia personal para la vida independiente y la promoción de la autonomía de las personas con diversidad funcional (discapacidad) en España. Departamento de Filosofía y Filosofía Moral y Política (UNED)-Foro de Vida Independiente. Disponible en: https://uplalaunion.org/wpcontent/uploads/2020/04/Asistente_personal.pdf

Allué, M. (2005). Discapacitados: Ia reivindicación de la igualdad en la diferencia. Barcelona: Bellaterra.

Artiaga, A. (2015). Producción política de los cuidados y de la dependencia: políticas públicas y experiencias de organización social de los cuidados (Tesis Doctoral inédita). Madrid, Universidad Complutense de Madrid.

Barnes, C. (1990). Cabbage Syndrome: The Social Construction of Dependence. Brighton: Falmer Press.

Bertaux, D. (2005). Los relatos de vida. Barcelona: Bellaterra.

Cerri, C. (2015). Dependencia y autonomía: una aproximación antropológica desde el cuidado de los mayores. Athenea Digital, 15(2), 111-140.

Conrad, P. (1982). Sobre la medicalización de la anormalidad y el control social. En D. Ingleby (Ed.), Psiquiatría Crítica. La política de la salud mental (pp. 129-154). Barcelona: Grijalbo. 
Dri, R. (2002). Racionalidad, sujeto y poder. Irradiaciones de la fenomenología del espíritu. Buenos Aires: Biblos.

Elias, N. (1990). La sociedad de los individuos. Barcelona: Península.

Evans, J. (2001). Independent Living and Centers for Independent Living as an Alternative to Institutions. Bruxelles: EDF AGA. Disponible en: https://disability-studies.leeds.ac.uk/ wp-content/uploads/sites/40/library/evans-Centres-Independent-Living-AlternativeInstitutions.pdf

Ferrante, C. (2014). Renguear el estigma. Cuerpo, deporte y discapacidad motriz. Buenos Aires, 1950-2010. Buenos Aires: Biblos.

Fisher, B., y Tronto, J. (1990). Toward a Feminist Theory of Caring. En E. Abel y M. Nelson (Dirs.), Circles of Care (pp. 36-54). Albany, NY: SUNY Press.

Jefatura del Estado. (2006, 15 de diciembre). Ley 39/2006, de 14 de diciembre, de Promoción de la Autonomía Personal y Atención a las personas en situación de dependencia. Boletín Oficial del Estado n. ${ }^{\circ}$ 299. https://www.boe.es/eli/es/l/2006/12/14/39/con

Gómez Bueno, C. (2000). Del etiquetado de las ocupaciones según nivel de cualificación. Revista de Sociología del Trabajo, 39, 33-61.

Gómez Bueno, C., Bretin, H., y Navas, R. (2011). Anticoncepción en Andalucía II. Representaciones, discursos y prácticas de adolescentes y adultas, con y sin discapacidad. Sevilla: Consejería de Salud, J.A. Disponible en: http://hdl.handle.net/10481/17743

Gómez Bueno, C., y Martín Palomo, M.T. (en prensa). Tecno-cuidados en los hogares. Cualificaciones requeridas, activadas y activables en el Servicio de Ayuda a Domicilio. Cuadernos de Relaciones Laborales, 38.

Díaz Velázquez, E. (2010). Ciudadanía, identidad y exclusión social de las personas con discapacidad. Política y Sociedad, 47(1), 115-135.

Grassi, E., Hintze, S., y Neufeld, M. (1994). Politicas sociales, crisis y ajuste estructural: un análisis del sistema educativo, de obras sociales y de las políticas alimentarias. Buenos Aires: Espacio Editorial.

Hochschild, A. (1983). The Managed Heart: Commercialization of Human Feeling. Berkeley: University of California Press.

IMSERSO (2020). Información estadistica destacada del SAAD. Disponible en: http://www. imserso.es/imserso_01/documentacion/estadisticas/info_d/index.htm

Kagitcibasi, C. (2005). Autonomy and Relatedness in Cultural Context: Implications for Self and Family. Journal of Cross Cultural Psychology, 36(4), 403-422.

López González. M. (2008). Mujeres con discapacidad: Mitos y realidades en las relaciones de pareja y en la maternidad. Barcelona: Narcea.

Martín Palomo, M.T. (2010). Autonomía, dependencia y vulnerabilidad en la construcción de la ciudadanía. Zerbitzuan, 48, 57-69.

Martín Palomo, M.T. (2016). Cuidado, vulnerabilidad e interdependencias. Nuevos retos politicos. Madrid: CEPC. 
Martín Palomo, M.T., y Damamme, A. (en prensa). Presentación. Cuidados, en la encrucijada de la investigación. Cuadernos de Relaciones Laborales, 38.

Martín Palomo, M.T., Fernández-Mayoralas, G., y Prieto Flores, M.E. (2018). Potencialidades y fragilidades del envejecer. Prisma Social, 11, 511-520.

Martínez Buján, R. (2014). Los modelos territoriales de organización social del cuidado a personas mayores en los hogares. REIS, 145, 99-126.

Moscoso, M. (2016). Encorsetar la diferencia: la discapacidad como «valor». Daimon. Revista Internacional de Filosofía, 5, 909-915.

Moscoso, M., Martín Palomo, M. T., y Muñoz Terrón, J.M. (2014). Abusos, violencias y malos tratos en mujeres discapacitadas: una aproximación teórica y metodológica. En Actas del XIII Congreso de Antropología de la FEAA del EE (pp. 5576-5294). Tarragona: Universidad Rovira i Virgili.

Molinier, P. (2011). Antes que todo, el cuidado es un trabajo. En P. Molinier y L.G. Arango (Comps.), El trabajo y la ética del cuidado (pp. 45-64). Cali: La Carreta Social.

Molinier, P., y Legarreta, M. (2016). Subjetividad y materialidad del cuidado: ética, trabajo y proyecto político. Papeles del CEIC, 2016/1(presentación), 1-14.

Morris, J. (2001). Impairment and Disability: Constructing an Ethics of Care which Promotes Human Rights. Hypatia, 16(4), 1-16.

Muyor Rodríguez, J., y Minguela Recover, M.Á. (2019). Cuidados, vida independiente y diversidad funcional: Análisis teórico y vinculaciones prácticas. Revista de Treball Social, 216, 29-47. DOI: 10.32061/RTS2019.216.02

Paperman, P. (2011). La perspectiva del care: de la ética a lo político. En P. Molinier y L.G. Arango (Comps.), El trabajo y la ética del cuidado (pp. 25-44). Cali: La Carreta Social.

Palacios, A. (2008). El modelo social de discapacidad: origenes, caracterización y plasmación en la Convención Internacional sobre los Derechos de las Personas con Discapacidad. Madrid: Cinca.

Shakespeare, T. (2000). Help. Birmingham: Venture Press.

Thomas, C. (1993). De-constructing Concepts of Care. Sociology, 27(4), 649-669.

Thomas, C. (2007). Sociologies of Disability and IIIness: Contested Ideas in Disability Studies and Medical Sociology. Nueva York: Palgrave Macmillan.

Tobío, C., Agulló, M.S., Gómez, M.V., y Martín Palomo, M.T. (2010). El cuidado de las personas. Un reto para el siglo XxI. Barcelona: Fundación La Caixa.

Toboso, M., y Rogero, J. (2012). «Diseño para todos» en la investigación social sobre personas con discapacidad. REIS, 1(140), 163-172. DOI: 10.5477/cis/reis.140.163

Tronto, J. (1993). Moral Boundaries. A Political Argument for an Ethic of Care. London: Routledge.

Venturiello, M.P. (2016). La trama social de la discapacidad: cuerpo, redes familiares, y vida cotidiana. Buenos Aires: Biblos. 